\title{
146. Note on a Theorem for Metrizability
}

\author{
By Jun-iti NAGATA \\ Department of Mathematics, Osaka City University \\ (Comm. by K. Kunugi, M.J.A., Dec. 12, 1957)
}

In the present note, we shall apply the previous metrization theorem $^{1)}$ to an open problem and shall prove the metrizability of a $T_{1}$-space $X$ satisfying the following condition of T. Inagaki: ${ }^{2)}$

For every point $p$ of $X$, we can assign a nbd (=neighborhood) basis $\left\{V_{n}(p) \mid n=1,2 \cdots\right\}$ such that

I) for every $p \in X$ and $n$, there exists $m=\alpha(p, n)$ such that $p \in V_{m}(q)$ implies $V_{m}(q) \subseteq V_{n}(p)$,

II) for every $p \in X$ and $n$, there exists $l=\beta(p, n)$ such that $q \in V_{l}(p)$ implies $p \in V_{n}(q)$.

Theorem. In order that a $T_{1}$-space $X$ is metrizable it is necessary and sufficient that $X$ satisfies the above condition.

Proof. Since the necessity is clear, we prove only the sufficiency.

1. First, we remark that we can assume, without loss of generality, that $m<n$ implies $V_{m}(p) \supseteq V_{n}(p)$ for every $p \in X$; otherwise we have the fulfilment of the condition by replacing $V_{n}(p)$ with $V_{1}(p)_{\wedge} \cdots V_{n}(p)$.

2. For every $p \in X$ and $n$, we can choose $k=\gamma(p, n)$ such that $q \in V_{k}(p)$ implies $p \in V_{m}(q) \subseteq V_{n}(p)$ for $m=\alpha(p, n)$.

To show this, let $m=\alpha(p, n), k=\beta(p, m)=\gamma(p, n)$. Then $q \in V_{k}(p)$ implies $p \in V_{m}(q) \subseteq V_{n}(p)$ by I) and II).

3. For every $p \in X$ and $n$, there exist nbds $M_{n}^{1}(p)$ and $M_{n}^{2}(p)$ of $p$ such that $q \notin V_{n}(p)$ implies $M_{n}^{1}(p) \frown M_{n}^{2}(q)=\phi$.

We let $\quad k=\gamma(p, n), \quad l=\beta(p, n), \quad k^{\prime}=\gamma(p, l)$;

$$
V_{k}(p)=M_{n}^{1}(p), \quad V_{k^{\prime}}(p)=M_{n}^{2}(p) .
$$

Now, let $q \notin V_{n}(p), r \in M_{n}^{1}(p) \frown M_{n}^{2}(q) \neq \phi$.

Then in the case of $m=\alpha(p, n) \leqq \alpha(q, l)=m^{\prime},{ }^{3)}$ we have

$$
q \in V_{m^{\prime}}(r) \subseteq V_{m}(r) \subseteq V_{n}(p)
$$

from 2, which contradicts $q \notin V_{n}(p)$.

In the case of $m=\alpha(p, n) \geqq \alpha(q, l)=m^{\prime}$, we have

$$
p \in V_{m}(r) \subseteq V_{m^{\prime}}(r) \subseteq V_{l}(q),
$$

1) J. Nagata: A theorem for metrizability of a topological space, Proc. Japan Acad., 33, no. 3 (1957), Theorem 1. See, also, J. Nagata: A contribution to the theory of metrization, Jour. Inst. Polytech., Osaka City Univ., 8, no. 2 (1957).

2) T. Inagaki: Sur les espaces à structure uniforme, Jour. of the Faculty of Sciences, Hokkaido University, 10 (1943). Prof. Inagaki proved in the paper that a separable space satisfying this condition was perfectly separable. We have learned from Prof. K. Morita that the metrization of such a space is an open problem.

3) We remark that this $l$ does not mean $\beta(p, n)$ but $\beta(q, n)$. 
which contradicts $q \notin V_{n}(p)$ by II). Therefore we conclude $M_{n}^{1}(p) \frown$ $M_{n}^{2}(q)=\phi$.

4. For every $p \in X$ and $n$, there exist nbds $N_{n}^{1}(p)$ and $N_{n}^{2}(p)$ such that $q \in N_{n}^{1}(p)$ implies $N_{n}^{2}(q) \subseteq U_{n}(p)=S\left(p, \mathfrak{B}_{n}\right),{ }^{4)}$ where $\mathfrak{B}_{n}=\left\{V_{n}(x) \mid x \in X\right\}$.

To show this, we let

$$
j=\gamma^{\prime}(p, n)=\operatorname{Max}(\alpha(p, n), \gamma(p, n)) .
$$

Then we see from 2 that $p \in V_{j}(q)$ implies $V_{j}(q) \subseteq V_{n}(p)$ and that $q \in V_{j}(p)$ implies $V_{j}(q) \subseteq V_{n}(p)$. Next, we let

$$
N_{n}^{1}(p)=S\left(p, \mathfrak{F}_{n}^{\prime}\right), \text { where } \mathfrak{V}_{n}^{\prime}=\left\{V_{j}(x) \mid j=\gamma^{\prime}(x, n), x \in X\right\},
$$

$N_{n}^{2}(p)=V_{j}(p)$, where $j=\gamma^{\prime}(p, n)$. If $q \in N_{n}^{1}(p)$, then there exists $x \in X$ satisfying $p, q \in V_{j}(x), j=\gamma^{\prime}(x, n)$.

In the case of $j=\gamma^{\prime}(x, n) \leqq \gamma^{\prime}(q, n)=j^{\prime}$, we have

$$
N_{n}^{2}(q)=V_{j^{\prime}}(q) \subseteq V_{j}(q) \subseteq V_{n}(x)
$$

from $q \in V_{j}(x)$. Since $p \in V_{n}(x)$ is clear, this implies

$$
U_{n}(p)=S\left(p, \mathfrak{B}_{n}\right) \supseteq V_{n}(x) \supseteq N_{n}^{2}(q) .
$$

In the case of $j=\gamma^{\prime}(x, n) \geqq \gamma^{\prime}(q, n)=j^{\prime}$, we have

$$
p \in V_{j}(x) \subseteq V_{j^{\prime}}(x) \subseteq V_{n}(q)
$$

from $q \in V_{j^{\prime}}(x)$. Hence $U_{n}(p)=S\left(p, \mathfrak{B}_{n}\right) \supseteq V_{n}(q)$, and hence

$$
N_{n}^{2}(q)=V_{j^{\prime}}(q) \subseteq V_{n}(q) \subseteq U_{n}(p) .
$$

5. Using the result of 3 , we let

$$
\mathfrak{M}_{n}=\left\{M_{n}^{1}(x) \mid x \in X\right\} ; \quad S\left(p, \mathfrak{M}_{n}\right)=T_{n}^{1}(p), \quad M_{n}^{2}(p)=T_{n}^{2}(p) .
$$

Then $q \notin U_{n}(p)\left(=S\left(p, \mathfrak{B}_{n}\right)\right)$ implies $T_{n}^{2}(q) \frown T_{n}^{1}(p)=\phi$. Using the result of 4 , we let

$$
S_{n}^{1}(p)=N_{n}^{1}(p) \frown T_{n}^{1}(p), \quad S_{n}^{2}(p)=N_{n}^{2}(p) \frown T_{n}^{2}(p) .
$$

Then we have nbds $U_{n}(p), S_{n}^{1}(p), S_{n}^{2}(p)$ for every point $p$ of $X$ such that

i) $\left\{U_{n}(p) \mid n=1,2 \cdots\right\}$ is a nbd basis of $p$,

ii) $q \notin U_{n}(p)$ implies $S_{n}^{2}(q) \frown S_{n}^{1}(p)=\phi$,

iii) $q \in S_{n}^{1}(p)$ implies $S_{n}^{2}(q) \subseteq U_{n}(p)$.

Therefore we conclude the metrizability of $X$ by our previous theorem. ${ }^{5)}$

Corollary. In order that a $T_{1}$-space $X$ is metrizable it is necessary and sufficient that we can assign a sequence $\left\{V_{n}(p) \mid n=1,2 \cdots\right\}$ of nbds of every point $p$ of $X$ such that $\overbrace{n=1}^{\infty}\left(\smile V_{p \in A} V_{n}(p)\right)=\overbrace{n=1}^{\infty} S\left(A, \mathfrak{F}_{n}\right)=\bar{A}^{6)}$ for every subset $A$ of $X$, where $\mathfrak{B}_{n}=\left\{V_{n}(p) \mid p \in X\right\}$.

Proof. The necessity is obvious. Let $X$ be a $T_{1}$-space satisfying this condition and let $U(p)$ be an arbitrary nbd of an arbitrary point $p$ of $X$, then $p \notin \bar{X}-U(p)$. Hence $p \notin S\left(X-U(p), \mathfrak{B}_{n}\right)$ for some $n$, i.e. $S\left(p, \mathfrak{B}_{n}\right) \subseteq U(p)$. Hence $\left\{V_{n}(p) \mid n=1,2 \cdots\right\}$ is a nbd basis of $p$. Since

4) $S\left(p, \mathfrak{B}_{n}\right)={ }^{\cup}\left\{V \mid p \in V \in \mathfrak{B}_{n}\right\}$.

5) Loc. cit.

6) $S\left(A, \mathfrak{B}_{n}\right)=\cup\left\{V \mid V \cup A \neq \phi, V \in \mathfrak{B}_{n}\right\}$. 
$V_{n}(p)$ is a nbd of $p$, we can assign $m=\alpha(p, n)$ such that $S\left(p, \mathfrak{B}_{m}\right) \subseteq$ $V_{n}(p)$. Then $p \in V_{m}(q)$ implies $V_{m}(q) \subseteq V_{n}(p)$.

To show the fulfilment of II) by $\left\{V_{n}(p)\right\}$, we assume the contrary: $q_{i} \in V_{i}(p), \quad p \notin V_{n}\left(q_{i}\right)(i=1,2 \cdots)$ for definite $p \in X$ and $n$. Let $A=\left\{q_{i} \mid i=1,2 \cdots\right\}$, then $p \in \bar{A}$ and $p \notin \smile\left\{V_{n}(q) \mid q \in A\right\}$, which is a contradiction. Thus we conclude the validity of this corollary by the theorem. 